\title{
High-speed Depth Stream Generation from a Hybrid Camera
}

\author{
Xinxin Zuo ${ }^{\dagger, \ddagger} \quad$ Sen Wang ${ }^{\dagger, \ddagger} \quad$ Jiangbin Zheng ${ }^{\dagger} \quad$ Ruigang Yang ${ }^{\ddagger}$ \\ ${ }^{\dagger}$ Northwestern Polytechnical University, Xi'an, P.R.China \\ $¥$ University of Kentucky, Lexington, KY, USA \\ \{xinxinzuo2353,wangsen1312\}@gmail.com, zhengjb@nwpu.edu.cn, ryang@cs.uky.edu
}

\begin{abstract}
High-speed video has been commonly adopted in consumergrade cameras, augmenting these videos with a corresponding depth stream will enable new multimedia applications, such as 3D slow-motion video. In this paper, we present a hybrid camera system that combines a high-speed color camera with a depth sensor, e.g. Kinect depth sensor, to generate a depth stream that can produce both high-speed and high-resolution RGB+depth stream. Simply interpolating the low-speed depth frames is not satisfactory, where interpolation artifacts and lose in surface details are often visible. We have developed a novel framework that utilizes both shading constraints within each frame and optical flow constraints between neighboring frames. More specifically we present (a) an effective method to find the intrinsics images to allow more accurate normal estimation; and (b) an optimization-based framework to estimate the high-resolution/high-speed depth stream, taking into consideration temporal smoothness and shading/depth consistency. We evaluated our holistic framework with both synthetic and real sequences, it showed superior performance than previous state-of-the-art.
\end{abstract}

\section{Keywords}

High-speed imaging; depth stream; Intrinisic decomposition; shape from shading

\section{INTRODUCTION}

With the recent advances in depth sensing, active depth sensors have become to be widely used in many multimedia applications, from 3D TV, gaming, to virtual reality. Nevertheless depth sensors still suffer from limited resolution and refresh rate. There are some previous works that focus on improving the spatial resolution of the depth map (e.g., [25, $27,2,33])$. These methods have shown good performance in resolution enhancement. In this paper we pay our attention to the temporal resolution, i.e., the frame rate of depth sensing. High-speed video has been commonly adopted in

Permission to make digital or hard copies of all or part of this work for personal or classroom use is granted without fee provided that copies are not made or distributed for profit or commercial advantage and that copies bear this notice and the full citation on the first page. Copyrights for components of this work owned by others than ACM must be honored. Abstracting with credit is permitted. To copy otherwise, or republish, to post on servers or to redistribute to lists, requires prior specific permission and/or a fee. Request permissions from permissions@ acm.org.

MM '16, October 15-19, 2016, Amsterdam, Netherlands

(c) 2016 ACM. ISBN 978-1-4503-3603-1/16/10 . \$ $\$ 15.00$

DOI: http://dx.doi.org/10.1145/2964284.2964305 consumer-grade cameras and even cellphones. They allow the capture of high-speed motion that is difficult to observe with naked eyes. Augmenting these videos with a corresponding depth stream will enable new applications, such as high-speed motion analysis or sports video playback and high-quality gait analysis for medical purpose.

Among all the publicly available commodity depth sensors, the SwissRanger ${ }^{T M}$ and $\mathrm{PMD}^{\mathrm{TM}}$ can capture the depth at higher speed than $30 \mathrm{~Hz}$, but with a much lower resolution at about $100 \times 200$. For the well known Kinect depth sensor, it has depth resolution at $640 \times 480$ for Kinect version 1 and $512 \times 424$ for Kinect version 2 . Both have a refresh rate of $30 \mathrm{~Hz}$. No single active depth sensor can provide both reasonable depth resolution and fast update rate of at least $60 \mathrm{~Hz}$. To overcome this limitation, we present a hybrid camera system that combines a high-speed color camera with a Kinect depth sensor that, with our novel post-processing algorithm, can generate the depth stream that has the same frequency and resolution as the color camera.

Given our hybrid camera setup, one straightforward way to generate high-speed depth stream is to apply bi-directional interpolation based on the optical flow. This is easy to implement. However, it is not always sufficient, since the linear motion assumption is not always true. In fact, usually it is the non-linear motion between frames that makes high-speed video interesting.

In addition to the flow field, shading information also provides useful cues for depth stream generation. We present a novel algorithm that enforces the shading constraints with the flow guidance in a unified framework. Instead of recovering the depth sequence frame by frame, we formulate an objective function with the shading constraints within frames and optical flow constraints between frames. The objective function is solved in a hierarchical manner with coarse-to-fine strategy to generate all the unknown depth maps at once.

We are certainly not the first to explore the shading cue for depth recovery. In addition to classic shape-from-shading (SfS) work [4, 12], there are several sensor fusion papers (e.g. [24, 32]) that combine the raw noisy depth with shading constraints to enhance the initial depth, which can resolve the ambiguity in traditional SfS to some extent. But in our case, we do not even have a depth map to start the refinement process. The interpolated depth map from the flow information can be far away from the real surface. We are dealing with a more challenging sensor fusion problem here.

One of the prerequisites to apply SfS techniques is the 

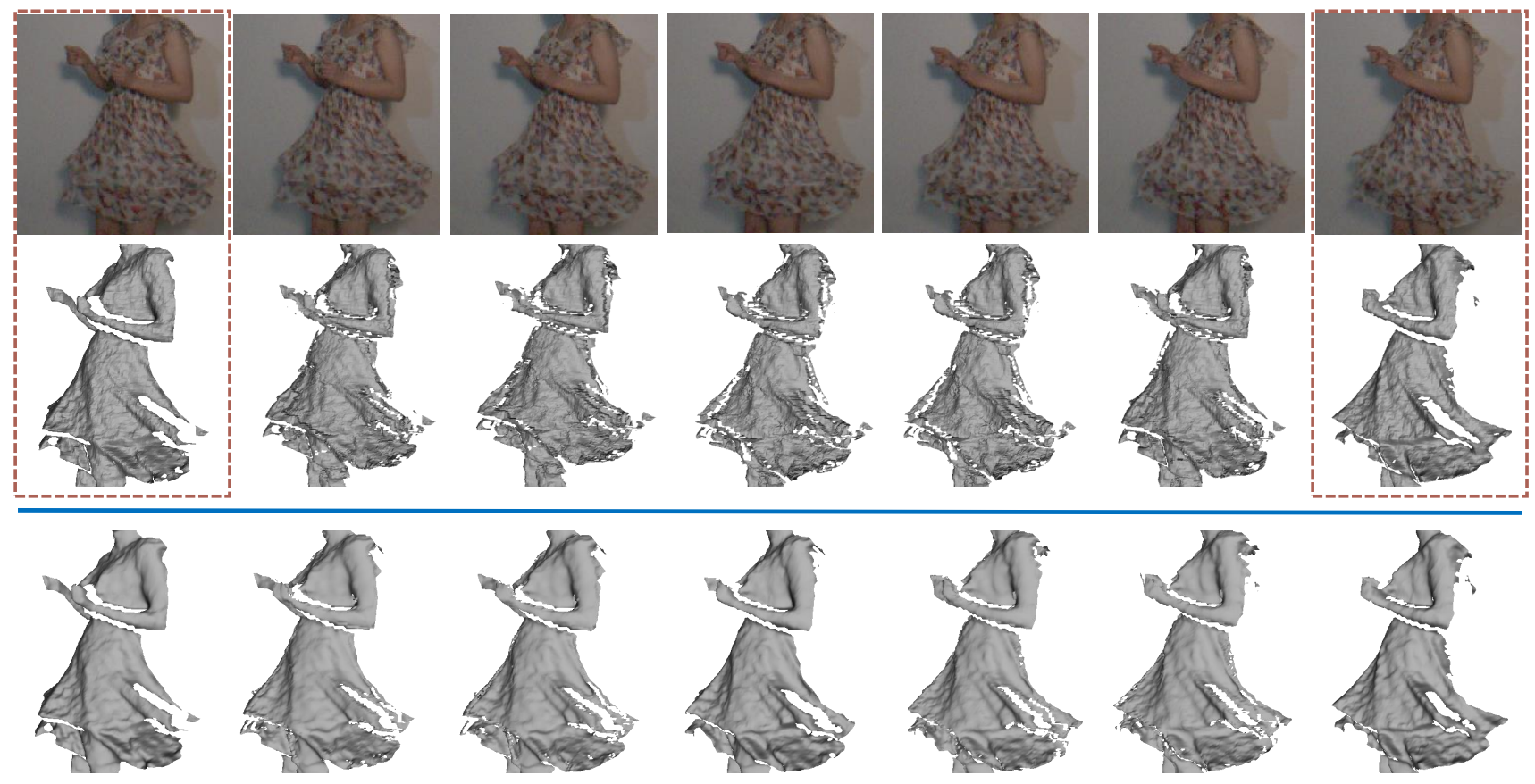

Figure 1: Depth stream results. (top) high-speed color images. (middle) interpolated depth maps based on optical/scene flow. The first and the last depth map are input. (bottom) corresponding depth generated with our algorithm.

known surface albedo. In our setup, we do not assume a known or uniform albedo. Therefore we must recover the surface albedo from captured images. This is the intrinsic image decomposition problem in computer vision, which is known to be ill-posed. We therefore develop a novel method that takes advantage of the fact that we do have depth maps for some of the color images. Our method compares favorably to previous state of the art in intrinsic image decomposition.

In summary the major contributions of this paper are:

- We identify a new problem that has not been studied, which is the low temporal refresh rate of currently available commodity depth sensors. Given the computational cost and their principles of operations, this limitation is unlikely to be addressed in hardware in the foreseeable future. We present a low-cost hybridcamera setup to overcome this limitation.

- Intrinsic image decomposition: SfS performs best when the surface albedo is uniform or known. We propose a new intrinsic decomposition method (Section 4.2) Compared to existing intrinsic images decomposition techniques, we have a few frames with known depth maps, but not all of them. We take advantages of these depth maps and provide a sequence-based optimization framework to estimate surface albedo, making the problem more tractable.

- Global optimization-based depth map interpolation: We formulate a novel optimization-based framework (Section 4.3), which combines the shading constraints in each frame and motion field constraints to generate the missing depth maps between know depth maps that are captured at low speed. An effective coarse-to-fine strategy is developed to make the optimization efficient and effective.

Our algorithm is validated with both synthetic and real datasets. In both cases satisfactory results have been obtained. Some examples are shown in Figure 1.

\section{RELATED WORK}

In this section, we will review the previous works in three related topics: hybrid camera setup, intrinsic image decomposition, and depth enhancement using shading information.

\subsection{Hybrid camera}

A single image system often cannot satisfy the diverse, sometime even contradictory, requirements of many imaging applications. Various types of hybrid camera setups have been developed to combine multiple image sensors to achieve better performance, such as high resolution, high speed, or high dynamic range. A high-speed color camera with low resolution (HSLR) and a low-speed color camera with high resolution (LSHR) is a common setup to deal with the motion blur in color images and recover high-speed color images with high resolution [29].

With the availability of video-rate depth cameras, researchers have developed techniques for depth image enhancement and super-resolution using a hybrid of depth camera and color cameras. For example, Zhu et al. [38] demonstrated improved accuracy and robustness on depth sensing with adaptive fusion of time-of-flight sensor and a pair of stereo cameras. Alternatively, the combination of high quality or high resolution color camera with a depth sensor is also a popular setup for depth map enhancement [33, 13]. For the most of these methods, they rely on the co-occurrence of depth and intensity discontinuities or they try to exploit the 
statistical relationship between these two modal images. In this paper, we present a novel hybrid setup with high-speed color camera and a depth sensor, e.g. Kinect depth sensor, to recover depth frames at the same frequency as the high-speed camera.

\subsection{Intrinsic image decomposition}

The problem of intrinsic image decomposition, which is to separate reflectance and illumination from a single image, is introduced by Barrow [6]. It is an ill-posed problem since there are two unknowns for every observation, additional constraints must be adopted to make the problem solvable. The Retinex theory [22] is widely used for this purpose. It assumes that shading variations are mostly low frequency while albedo changes are mostly high-frequency. Based on this assumption, many approaches have been proposed. For example, Tappen et al. [30] train a classifier from reflectance and shading datasets to separate these two components. Zhao [37] added non-local smoothness term on reflectance to preserve global shading and reflectance structure. Global priors, such the reflectance sparsity, are developed [14] and they perform clustering on image reflectance. $\mathrm{Bi}$ [7] also exploits the global sparsity cues by enforcing an L1 smoothness term. Barron [4] obtain strong priors by training from reflectance and shading exemplers and they try to recover the reflectance, shape and illumination in a unified framework.

All of these above-mentioned methods focus on a singleimage input. To reduce the ambiguity, other additional information are adopted. For example, image sequences [20, 21] are used under different lighting conditions. Also, depth images are important cues to at least reduce the ambiguities [11, 19, 5]. Lee et al. [23] simply adjusted the weight of shading constraints based on pixel normals, while they employed temporal constraints for RGBD video. Some researchers $[9,34,8]$ perform the decomposition in an interactive way with manual annotation as guidance. In our setup we have a video sequence with depth maps in some key frames. Therefore we have developed a new method to handle our unique input.

\subsection{Shape-from-shading and sensor fusion}

The SfS problem has long been studied since the pioneering work by Horn [18]. There are various regularization terms or prior assumptions [3, 4] that have been enforced to deal with the inherently ill-posed problem in traditional SfS. As it is really hard to solve this inverse rendering optimization, this has led to the work on photometric stereo [16, 36 ] that uses multiple images of a scene taken under different controlled illumination to compute the surface normal as a well-defined problem.

Recent methods have shown that SfS can be used to refine the noisy depth map captured from RGB-D cameras under uncontrolled lighting $[15,35]$. The rough normal computed from initial depth map provides the information to estimate the light source and also recover the multiple albedos from the color image $[24,32]$, which eliminates the need for assumptions and constraints on shape or the use of several images. This type of depth refinement has worked very well to provide more surface details. There are also previous works that adopt the shading constraints to refine the coarse $3 \mathrm{D}$ shape reconstructed using multi-view stereo [31].

All existing shading-refinement schemes assume that the initial depth is given for every image and the shading information is incorporated with the depth information to recover subtle surface details and remove noise. In our setup we do not have the initial depth map to start with. We also demonstrated that simply combining depth interpolation and shading refinement is unable to yield satisfactory results. Therefore we develop a new global optimization framework that considers shading constrains and optical flow constraints at the same time.

\section{SYSTEM SETUP}

We now briefly introduce our system setup and the overall processing pipeline. Our hybrid camera uses a PointGrey ${ }^{T M}$ Dragonfly camera as the high-speed color camera. It captures images with resolution $640 \times 480$ at $180 \mathrm{fps}$. We put the depth camera, for which we use the Kinect depth sensor, next to the PointGrey camera (see section 1 in supplementary material for more details). The original depth stream is captured at 30fps. The two cameras are calibrated and synchronized with system timestamps.

An overview of our high-speed depth map generation framework is shown in Figure 2. First, we will obtain the flow in-

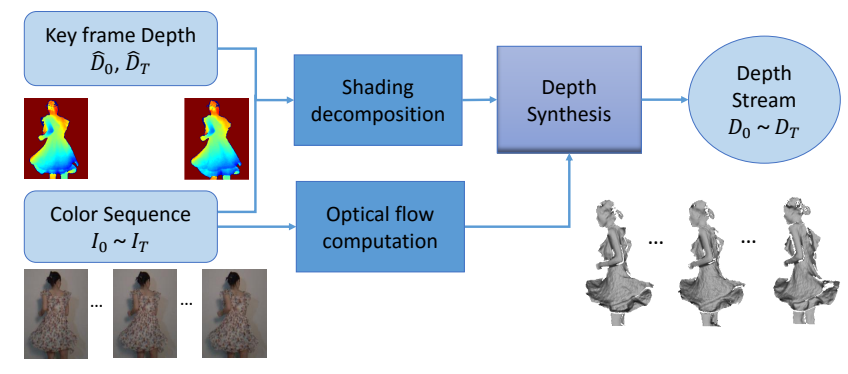

Figure 2: System Pipeline.

formation between color images. In the meantime the lighting condition is estimated in these key frames, which is assumed to be changing slowly over time. Then the albedo and shading images for the whole sequence are estimated with a novel decomposition algorithm. It uses the depth cues in key frames and temporal constrains (Section 4.2). Finally, the shading cues and flow information are combined together into our proposed global optimization framework, from which we can generate the depth stream $\left(D_{0} \sim D_{T}\right)$ between any two key frames (Section 4.3), leading to an output RGB-plus-depth stream at $180 \mathrm{~Hz}$ (the same rate as the high-speed color camera). Notice that we do not attempt to increase the spatial resolution of the depth map. Existing algorithms can be adopted if desired.

\section{OUR APPROACH}

Suppose we have two depth frames $\hat{D}_{0}$ and $\hat{D_{T}}$, which are denoted as the depth key frames, and color frames $I_{0} \sim I_{T}$, where $I_{0}$ and $I_{T}$ correspond to the depth frame $\hat{D_{0}}$ and $\hat{D_{T}}$ respectively. Our goal is to estimate or refine the depth frames $D_{0} \sim D_{T}$.

\subsection{Preprocessing}

First, we will describe some pre-processing steps and clarify some notations.

Depth warp The two cameras are calibrated and we warp 
the key frame depth maps into color coordinates using the calibrated intrinsic and extrinsic parameters.

3D point Suppose we have a pixel $p=(i, j)$ with depth $D(p)$, then with the depth camera intrinsic $K$ we can get its $3 \mathrm{D}$ point position as

$$
\begin{aligned}
X(p) & =D(p) K^{-1}(i, j, 1)^{T}, \\
K & =\left(\begin{array}{ccc}
f_{x} & 0 & \mu \\
0 & f_{y} & \nu \\
0 & 0 & 1
\end{array}\right)
\end{aligned}
$$

where $f_{x}$ and $f_{y}$ are the focal length in $x$ and $y$ direction, $\mu$ and $\nu$ are the camera's principal point.

Normal In this paper, we use the perspective camera projection model, and the unnormalized normal $\tilde{\boldsymbol{n}}_{p}$ for pixel $p$ can be computed as,

$$
\tilde{\boldsymbol{n}}(p)=(X(i, j+1)-X(p)) \times(X(i+1, j)-X(p))
$$

Then substitute the 3D points in Eq. 3 with Eq. 2 and Eq. 1, the normal can be written as

$$
\tilde{\boldsymbol{n}}(p)=\frac{D(i+1, j) \cdot D(i, j+1)}{f_{x} \cdot f_{y}}\left(\begin{array}{c}
\frac{f_{x} \cdot(D(i, j+1)-D(p))}{D(i+1, j)} \\
\frac{f_{y} \cdot(D(i+1, j)-D(p))}{D(i, j+1)} \\
\frac{(\mu-j) \cdot(D(i, j+1)-D(p))}{D(i+1, j)}+\frac{(\nu-i) \cdot(D(i+1, j)-D(p))}{D(i, j+1)}-1
\end{array}\right)
$$

Finally, we can normalize it to $\boldsymbol{n}(p)$.

Optical flow We have the color sequence $\mathbf{I}=\left[I_{0}, I_{1}, \cdots I_{T}\right]$ and the corresponding mapping between any two neighboring frames can be obtained from optical flow [10]. $W_{t}(p)$ maps the pixel $p$ in frame $t$ to next frame $t+1$.

\subsection{Intrinsic decomposition}

Before enforcing the shading constraints for generating high-speed depth stream, we need to perform the intrinsic image decomposition to separate the shading effect from albedo and estimate the lighting condition using the depth key frames.

\subsubsection{Lighting estimation}

Similar to the majority of prior work on lighting estimation, we assume the object surfaces to be Lambertian, based on which Spherical Harmonics (SH) can be used to represent the incident lighting $L$ efficiently. We use the first nine $\mathrm{SH}$ basis functions (up to second order), which is a good approximation for Lambertian reflectance [26]. Then the reflected irradiance $I$ for each pixel $p$ can represented as

$$
\begin{gathered}
I(p)=A(p) \cdot S(p), \\
S(p)=\sum_{m=1}^{9} l_{m} H_{m}(\boldsymbol{n}(p)),
\end{gathered}
$$

where $A(p)$ is represented as albedo vectors for pixel $p$ which contains three channels, and $S(p)$ donates the scalar shading vaule for $p$. $l_{m}$ are the corresponding SH coefficients of incident lighting, $H_{m}\left(\boldsymbol{n}_{p}\right)$ represent the $\mathrm{SH}$ basis functions (see section 2 in supplementary material for more details).

Similar to [28], we assume that the pixels within the same super-pixel share the same albedo value, therefore we cluster the color image to $S N$ segments with super-pixel algorithm [1].

For every two neighboring key depth maps with their color images, we can get the $l_{m}$ by minimizing the energy function,

$$
E_{L}=\sum_{t \in\{0, T\}} \sum_{s n=1}^{S N} \sum_{p}\left\|A_{t}^{s n}(p) \sum_{m=1}^{9} l_{m} H_{m}\left(\boldsymbol{n}_{t}(p)\right)-I_{t}(p)\right\|^{2},
$$

where $S N$ is the number of superpixels segments of color images, $A_{t}^{s n}(p)$ means the albedo in frame $t$ for pixel $p \in$ $s n$th segments, which is approximated by the mean color of the superpixel.

We can solve this minimization by computing the mean albedo of the superpixels and lighting coefficients in an iterative way and the iteration starts by setting the albedo as mean color of pixels inside each superpixels. Since we assume that the lighting is changing slowly during the data capture, we use the same lighting coefficients $l_{m}$ for the sequence between two key frames.

\subsubsection{Shading and albedo computation}

The goal of shading computation is recovering the albedo $A(p)$ and shading $S(p)$ that best match the image $I(p)$. This is an essential step before SfS can be used. Different from the previous works on shading decomposition using RGBD images, we do not have depth images in every frame but only some key frames. On the other hand, the depth information in key frames provides us the valuable cues to resolve the ambiguity for single image decomposition. Therefore, we propose to compute the shading images for the sequence between any two key frames in a unified optimization making use of the depth cues in key frames and albedo consistency constraints along the sequence. The objective function is described below.

First, we have the data terms. The first is to match the image input. We operate in the logarithmic domain and $\hat{A}$, $\hat{S}$ and $\hat{I}$ stand for the logarithm of albedo, shading and color image, respectively.

$E_{d \_i m}=\sum_{t=0}^{T} \sum_{c \in\{R, G, B\}} \sum_{p} \omega_{t}^{\text {lum }}(p)\left\|\hat{A}_{t}(p, c)+\hat{S}_{t}(p)-\hat{I}_{t}(p, c)\right\|^{2}$,

where

$$
\omega_{t}^{\text {lum }}(p)=\operatorname{lum}(p)+\epsilon
$$

This term is weighted by the luminance of the input intensity image to prevent disproportionably strong affect of dark pixels.

The second data term is to preserve the initial shading images in key frames, which can be computed with the lighting vector(Section 4.2.1) and coarse surface normals. The generated shading images may not be accurate but they are good approximations.

$$
E_{d_{-} s}=\sum_{t \in\{0, T\}} \sum_{p}\left\|\log \left(\sum_{m=1}^{9} l_{m} H_{m}\left(\boldsymbol{n}_{p}\right)\right)-\hat{S}_{t}(p)\right\|^{2}
$$

Regularization terms Next, we have the spatial priors for shading and albedo in each frame, as well as the temporal consistency priors of albedo between neighboring frames.

The albedo smoothness term for each frame with adaptive weighting is

$$
E_{s_{-} a}=\sum_{t=0}^{T} \sum_{p, q \in \mathscr{N}} \omega_{t}^{a}(p, q)\left\|\hat{A}_{t}(p)-\hat{A}_{t}(q)\right\|_{2}^{2}
$$

The adaptive weight is computed with the differences of intensity and chromaticity between adjacent pixels 


$$
\omega_{t}^{a}(p, q)=\left\{\begin{array}{cc}
0 & \text { if } \nabla c h_{t}>\tau_{c h} \\
0 & \text { if } \nabla \text { lumt }>\tau_{\text {lum }} \\
\exp \left(-\frac{\nabla c h_{t}^{2}}{\sigma_{c h}^{2}}\right) \cdot \exp \left(-\frac{\nabla l u m_{t}^{2}}{\sigma_{\text {lum }}^{2}}\right) & \text { otherwise }
\end{array}\right.
$$

where

$$
\begin{aligned}
\nabla c h_{t} & =\left\|\operatorname{ch}\left(I_{t}(p)\right)-\operatorname{ch}\left(I_{t}(q)\right)\right\|_{2} \\
\nabla \operatorname{lum}_{t} & =\left\|\operatorname{lum}\left(I_{t}(p)\right)-\operatorname{lum}\left(I_{t}(q)\right)\right\|
\end{aligned}
$$

The shading smoothness term is formulated as below

$$
E_{s_{\_} s}=\sum_{t=0}^{T} \sum_{p, q \in \mathscr{N}} \omega_{t}^{n}(p, q)\left\|\hat{S}_{t}(p)-\hat{S}_{t}(p)\right\|^{2},
$$

For key frame 0 and $\mathrm{T}$, we have coarse depth from which we can compute the surface normal $\boldsymbol{n}$, and then the smooth weight $\omega_{t}^{n}(p, q)$ is set as

$$
\omega_{t}^{n}(p, q)=1-\exp \left(-\frac{\left(\boldsymbol{n}_{p}^{T} \boldsymbol{n}_{q}\right)^{2}}{\sigma_{n}^{2}}\right)
$$

It means that we will favor smooth shading for adjacent pixels that have similar normal orientations. For other frames $1 \sim T-1$ that only have color images, the smooth weight may be set as a constant value. However, the weight is not easy to choose. As shown in figure 3 , the texture is not separated clearly from shading image if the weight is small (figure 3(b)), while the shading is over-smoothed when the weight is large (figure 3(c)).

To deal with this problem, we decide to propagate the adaptive weighting terms computed in key frames into other frames. In more detail, the weight term in frame 0 is propagated forward to next frames using the following equation

$\omega_{t}^{n, f}(p, q)=\left\{\begin{array}{cc}\omega_{t-1}^{n, f}\left(p_{t-1}, q_{t-1}\right) & p_{t-1}, q_{t-1} \in \mathscr{N} \text { for } 1<t<T \\ \omega_{0}^{n}\left(p_{0}, q_{0}\right) & p_{0}, q_{0} \in \mathscr{N} \text { for } t=1 \\ 0.1 & \text { otherwise for } t \in[1, T-1]\end{array}\right.$

In the above formula, for adjacent pixels $p$ and $q$ in frame $t$, the corresponding pixels $p_{t-1}$ and $q_{t-1}$ in previous frame $t-1$ can be found with optical flow. The smooth weight is propagated from previous frames when the adjacent pixels stay connected as neighbours, otherwise is set to 0.1 . In this way, the smooth shading is propagated and shading details will still get preserved. We can compute the weight $\omega_{t}^{n, b}$ using backward propagation from frame $T$. The weight is blended as

$$
\omega_{t}^{n}(p, q)=\max \left\{\omega_{t}^{n, f}(p, q), \omega_{t}^{n, b}(p, q)\right\}
$$

The temporal albedo consistency is defined as

$$
E_{t_{-a}}=\sum_{t=0}^{T-1} \sum_{p} \omega_{t}^{f b}(p) \omega_{t}^{t a}(p)\left\|\hat{A}_{t}(p)-\hat{A}_{t+1}\left(W_{t}(p)\right)\right\|^{2}
$$

The weight terms are necessary as we need to prevent the artifacts caused by occlusion between frames and the inaccuracy in flow computation. The weighting term $\omega_{t}^{f b}(p)$ is used to discard the occluded regions and is set to 0 if the froward-backward flow consistency check fails, otherwise it is set to 1 . We set the weighting term $\omega_{t}^{t a}$ as

$$
\omega_{t}^{t a}(p)=\left\{\begin{array}{cc}
0 & \left\|I_{t}(p)-I_{t+1}\left(W_{t}(p)\right)\right\|_{2}>\tau_{t a} \\
1 & \text { otherwise }
\end{array}\right.
$$

The final objective function is the weighted sum of all terms, that is

$$
E_{l}=E_{d_{-} i m}+\lambda_{d_{-} s} \cdot E_{d_{-} s}+\lambda_{s_{-} a} \cdot E_{s_{-} a}+\lambda_{s_{-} s} \cdot E_{s_{-} s}+\lambda_{t_{-} a} \cdot E_{t_{-} a}
$$

The objective function can be solved with linear optimization.

With the above propagation framework, for middle frames that do not have depth images, we are still able to decompose the texture from color images while preserving shading details, as shown in figure $3(\mathrm{~d})$.
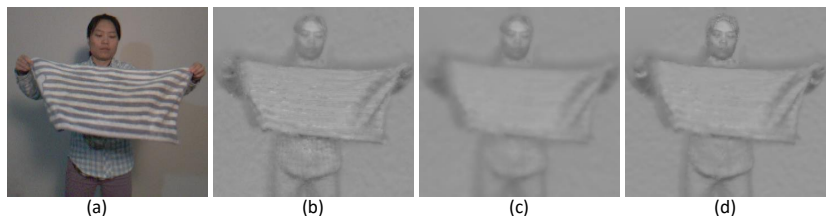

Figure 3: Results on intrinsic decomposition. (a) a sampled middle frame; (b) decomposed shading image when constant shading smooth weight $\omega_{t}^{n}$ set as 1.0; (c) shading image computed when $\omega_{t}^{n}$ is set as $6.0 ;(\mathrm{d})$ our shading image with adpative smooth shading weight.

\subsection{Depth stream generation framework}

Now we have all the albedo and shading images for each frame $\left(A_{0} \sim A_{T}\right.$ and $\left.S_{0} \sim S_{T}\right)$, and the key frame depth images $\left(\tilde{D}_{0}\right.$ and $\left.\tilde{D_{T}}\right)$, we can recover the in-between depth frames.

Given equally sampled temporal frames indexed by $t$, the $3 \mathrm{D}$ scene velocity , $U_{t}(D(p))$ at pixel $p$, is computed as (for simplicity, we omit $p$ here),

$$
U_{t}(\mathbf{D})=X_{W_{t}}\left(D_{t+1}\left(W_{t}\right)\right)-X\left(D_{t}\right)
$$

where $\mathbf{D}=\left[D_{0}, D_{1}, \cdots D_{T-1}\right]$. The above function is the velocity field for the sequence.

It is assumed that the motion between neighboring color frames should not be too fast and will prefer small velocities that are spatially smooth. Our first term is denoted as the velocity term,

$$
E_{v}=\sum_{t=0}^{T-1} \sum_{p}\left(\left\|U_{t}(D)\right\|^{2}+\lambda_{v} \psi\left(\left\|\nabla U_{t}(D)\right\|^{2}\right)\right)
$$

where

$$
\psi\left(a^{2}\right)=\sqrt{a^{2}+\varepsilon^{2}}
$$

The first term in Eq. 23 has the similar effect as bi-linear interpolation that will spread the spatial movement in $3 \mathrm{D}$ between the two key frames into the whole sequence. The second term in Eq. 23 is exploited here to preserve the smooth scene flow field [17]. We do not enforce any linear constraints for this term while only spatial smoothness is favored for the $3 \mathrm{~d}$ flow field. Therefore, the velocity term will try to preserve the smooth transition along the depth sequence while allowing for non-linear motion.

Secondly, with the albedo images computed from the previous section, we have the shading constraint that penalizes the differences between rendered images and the captured color images. We use the Charbonnier penalty function(Eq. 24), which is more robust to the outliers in the shading image,

$$
E_{s}=\sum_{t=0}^{T} \sum_{p} \psi\left(\left\|A_{t}(p) \sum_{m=1}^{9} l_{m} H_{m}(\boldsymbol{n}(p))-I_{t}(p)\right\|_{2}^{2}\right)
$$



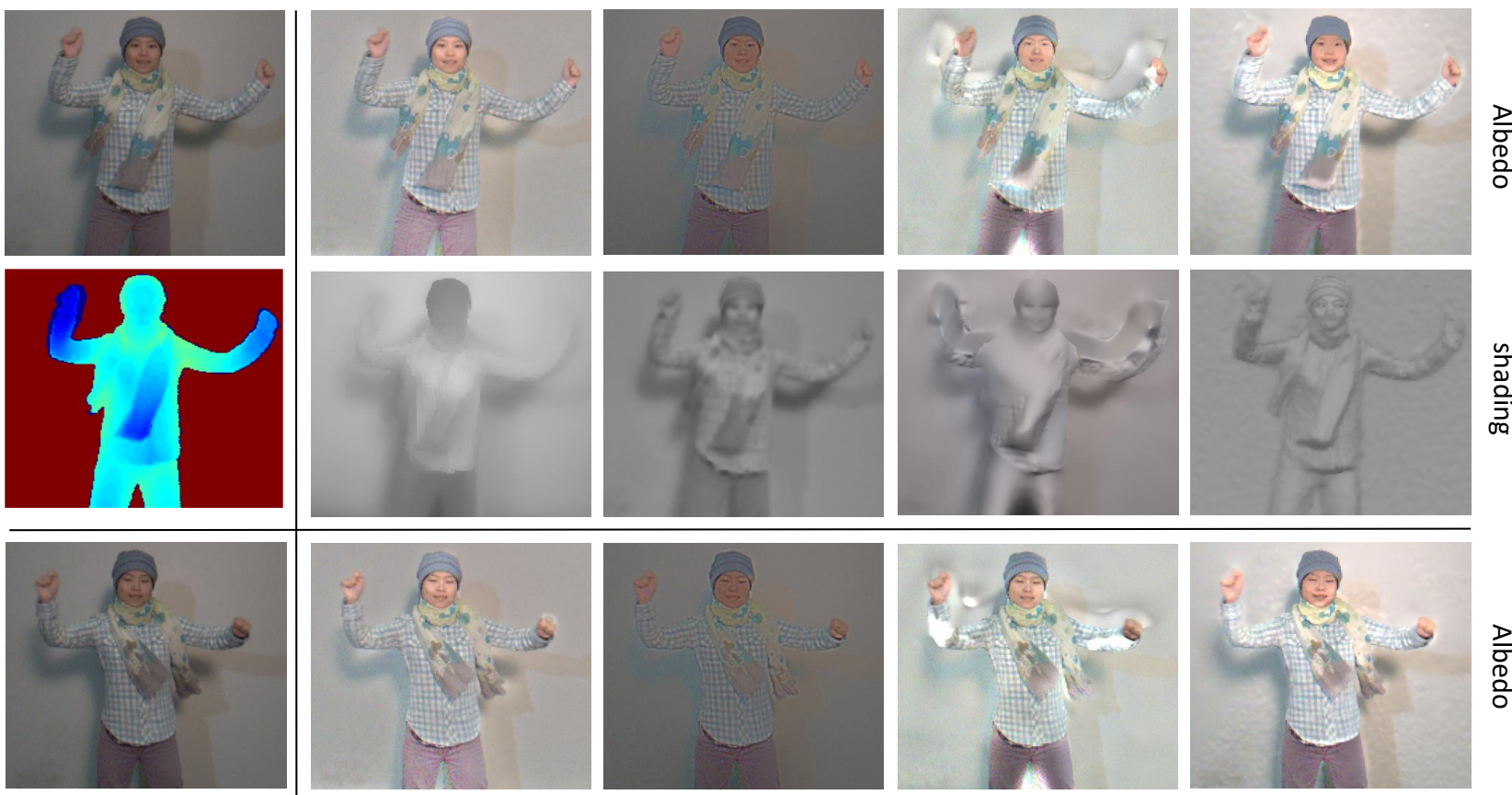

$\frac{\substack{n \\ \frac{2}{2}}}{\frac{n}{2}}$

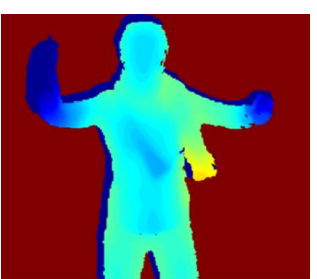

(a) input

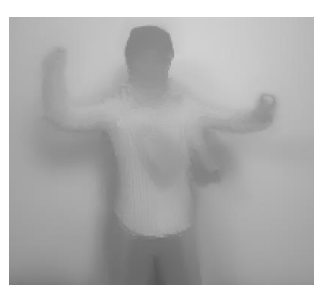

(b) Chen13[11]

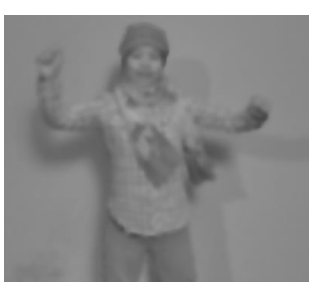

(c) Jeon14[19]

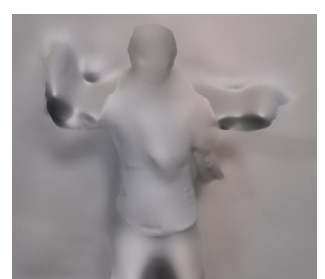

(d) Barron16[5]

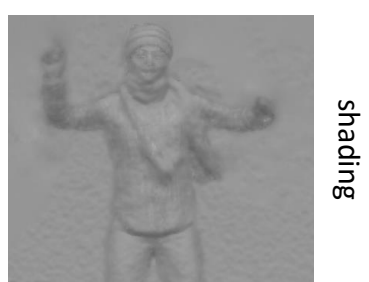

(e) ours

Figure 4: Results on sampled images from hand waving stream. (a) Input color image and depth map. (b-e) Albedo and Shading images estimated by three recent approaches for intrinsic decomposition and by our approach.

For the above formula, $\boldsymbol{n}(p)$ can be substituted with depth $D$ based on the normal computation equation (Eq. 4 ). Therefore, the shading constraints are directly enforced on depth streams.

Next, in order to have the smooth surface, we utilize a Laplacian smoothness term for each frame. We use the adaptive weighting again to preserve the surface boundary.

$$
E_{l a p}=\sum_{t=0}^{T} \sum_{p}\left(D_{t}(p)-\frac{\sum_{q \in \mathscr{N}_{p}} \omega_{t}(p, q) \cdot D_{t}(q)}{\sum_{q \in \mathscr{N}_{p}} \omega_{t}(p, q)}\right)^{2}
$$

In the above formula, the weighting term is computed using the Gaussian filter as,

$$
\omega_{t}(p, q)=\exp \left(-\frac{\left\|D_{t}(p)-D_{t}(q)\right\|^{2}}{\sigma_{\text {lap }}^{2}}\right)
$$

Finally, the data term enforces the constraint provided by two depth key frames $\tilde{D}_{0}$ and $\tilde{D}_{T}$, which can be regarded as the boundary condition.

$$
E_{d}=\sum_{t \in\{0, T\}} \sum_{p}\left(D_{t}(p)-\tilde{D}_{t}(p)\right)^{2}
$$

In summary, our energy function for depth map generation is formulated as

$$
E=E_{v}+\lambda_{s} \cdot E_{s}+\lambda_{l a p} \cdot E_{l a p}+\lambda_{d} \cdot E_{d}
$$

The formulation for generating the depth stream is a nonlinear minimization problem. We use the Levenberg-Marquard (LM) method to solve this optimization problem. We have found out that it is difficult to achieve convergence directly since the solution space could have many local minimums. We instead develop a coarse-to-fine refinement strategy. First, we build up the pyramid for shading sequence and two key depth frames. In our implementation, we have four layers for the pyramid. In the first two coarsest levels, the energy function Eq. 29 is minimized and we can get the initial rough depth sequence. Then we will refine the depth with shading constraints separately for each frame. In this step, the initial depth propagated from the coarser level will be enhanced with the shading constraints together with Laplacian smoothness with the Eq. 25 and Eq. 26.

\section{EXPERIMENTS}

We have captured some real data sets with the prototype of our hybrid camera setup described in Section 3 and tested our method on these datasets. Also, we have generated some synthetic datasets to validate the method quantitatively. In this section, we will show quantitative and qualitative comparison results. All the parameters in our algorithms are showed on Table 1, these values are tuned empirically and remain fixed for all the experiments.

We have also implemented two baseline algorithms. The 


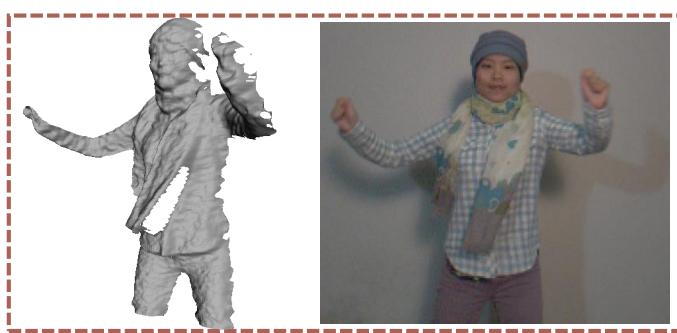

(a) Input key frame 0

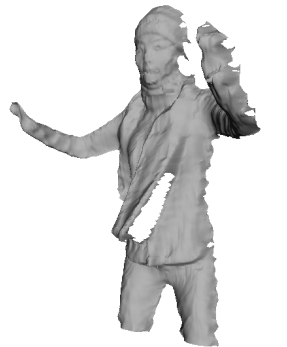

(d) Refined key frame 0

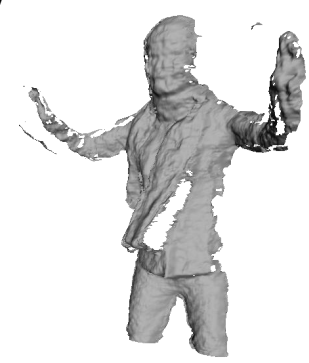

(e) BL

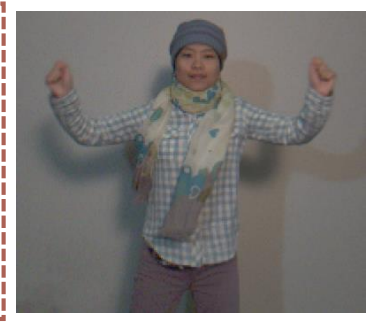

(b) Sampled middle frame

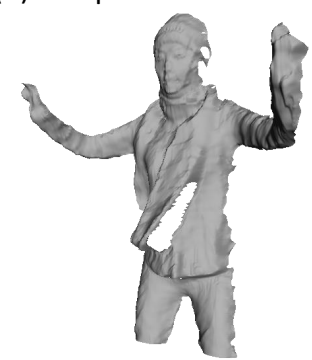

(f) ours

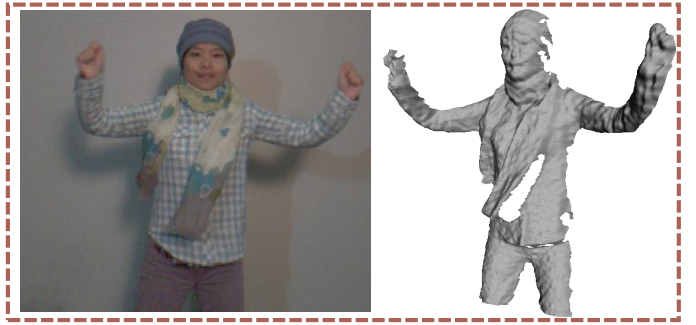

(c) Input key frame T

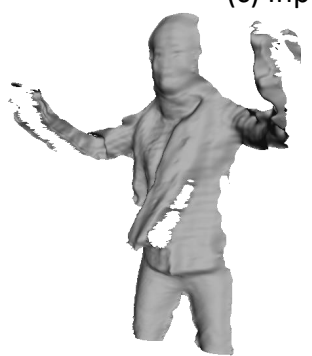

(g) SBL

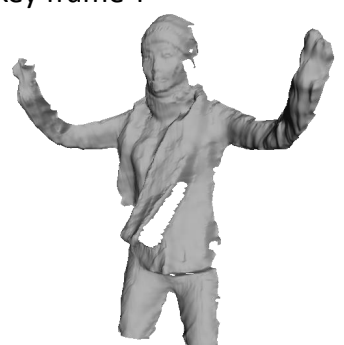

(h) Refined key frame T

Figure 5: Results on the hand waving sequence. To better show the shape of the hands, we adjust the perpective angle of the generated mesh. We have two key frame color images and the initial meshes as input shown in (a) and (c). (d) and (h) are the key frame meshes after refinement. The second row shows the interpolation result of one sampled frame shown in (b). (e)-(g) are the recovered meshes using BL, our proposed method and SBL, respectively.

\begin{tabular}{|c|c|c|c|c|c|c|c|}
\hline Eq. 7 & Eq. 9 & \multicolumn{4}{|c|}{ Eq. 12} & Eq. 16 & Eq. 20 \\
\hline$S N$ & $\epsilon$ & $\tau_{c h}$ & $\tau_{\text {lum }}$ & $\sigma_{c h}$ & $\sigma_{\text {lum }}$ & $\sigma_{n}$ & $\tau_{t a}$ \\
\hline 30 & 0.001 & 0.06 & 0.10 & 0.01 & 0.05 & 0.1 & 0.08 \\
\hline Eq. 24 & Eq. 27 & \multicolumn{3}{|c|}{ Eq. 21} & \multicolumn{3}{|c|}{ Eq. 29} \\
\hline$\varepsilon$ & $\sigma_{\text {lap }}$ & $\lambda_{S_{-} a}$ & $\lambda_{s_{-} s}$ & $\lambda_{t_{-} a}$ & $\lambda_{s}$ & $\lambda_{\text {lap }}$ & $\lambda_{d}$ \\
\hline 0.001 & $5 \mathrm{~mm}$ & 2.0 & 3.0 & 5.0 & 0.1 & 0.01 & 10 \\
\hline
\end{tabular}

image that is quite smooth, as shown in figure $4(\mathrm{~b})$. This method performs better on scene level decomposition not on surface details. Jeon et al. [19] explicitly deal with the texture in the image and separate the repeated texture patterns before shading decomposition. Therefore, the grid patterns can be separated into albedo image correctly, while the texture on the scarf stays at the shading image, as shown

Table 1: The parameter settings for our experiments.

first is to use the optical flow information to interpolate the in-between frames. We denote this baseline method as the BL (bi-directional interpolation) method. The second is to refine the interpolated depth map with shading information. More specifically, we first apply a low-pass filter to smooth out the interpolated depth maps and then do the refinement with shading constraints, e.g., using method [24], to recover more surface details. We denote this method as the SBL method.

\subsection{Intrinsic image decomposition}

We have compared our method with three previous works on intrinsic image decomposition with single RGB-D image as input. As shown in figure 4, the upper rows demonstrate the decomposition for key frame images that have captured depth frames as input. For the lower row, it corresponds to a sampled middle frame that has no captured depth as input. For this middle frame, the interpolated depth from the BL method is taken as the input depth for all these comparison methods; for our method the interpolated depth is not needed, since the decomposition is performed using global optimization with key frames and middle frames computed all together.

Chen et al. [11] performs the decomposition without taking surface details into account and tends to get shading in figure 4(c). Figure 4(d) displays the results using the method [5] which takes as input a single RGB-D image and produces as output an improved depth map, albedo image, shading image and illumination model. This approach is sensitive to the outliers in input depth map, especially along the surface boundary, as the zero depth value is not handled explicitly. It will fail in our case where the depth round the moving hands and arms is quite noisy caused by motion blur and also the interpolated depth is wrong around the arms. Figure 4(e) shows the results from our method where the texture is successfully decomposed into albedo image while real surface details, i.e. on the hat and the around the arms, can be seen in the shading image. More comparison results are shown in the supplementary material.

\subsection{Qualitative evaluation for depth frame gen- eration}

In Figure 5, we demonstrate the recovered depth of the relative simple case, where the human subject is waving her arms and hands quite fast. For this relative simple case, since the 3D motion can be approximated by the linear motion, the bi-directional interpolation(BL) result is acceptable in most of the parts except for some artifacts along the hands. After the shading refinement(SBL), the depth becomes more smooth and the surface details can be recovered. However, the artifacts have got passed from the interpolation result and the shading refinement could not handle this properly. In comparison with these two methods, our 


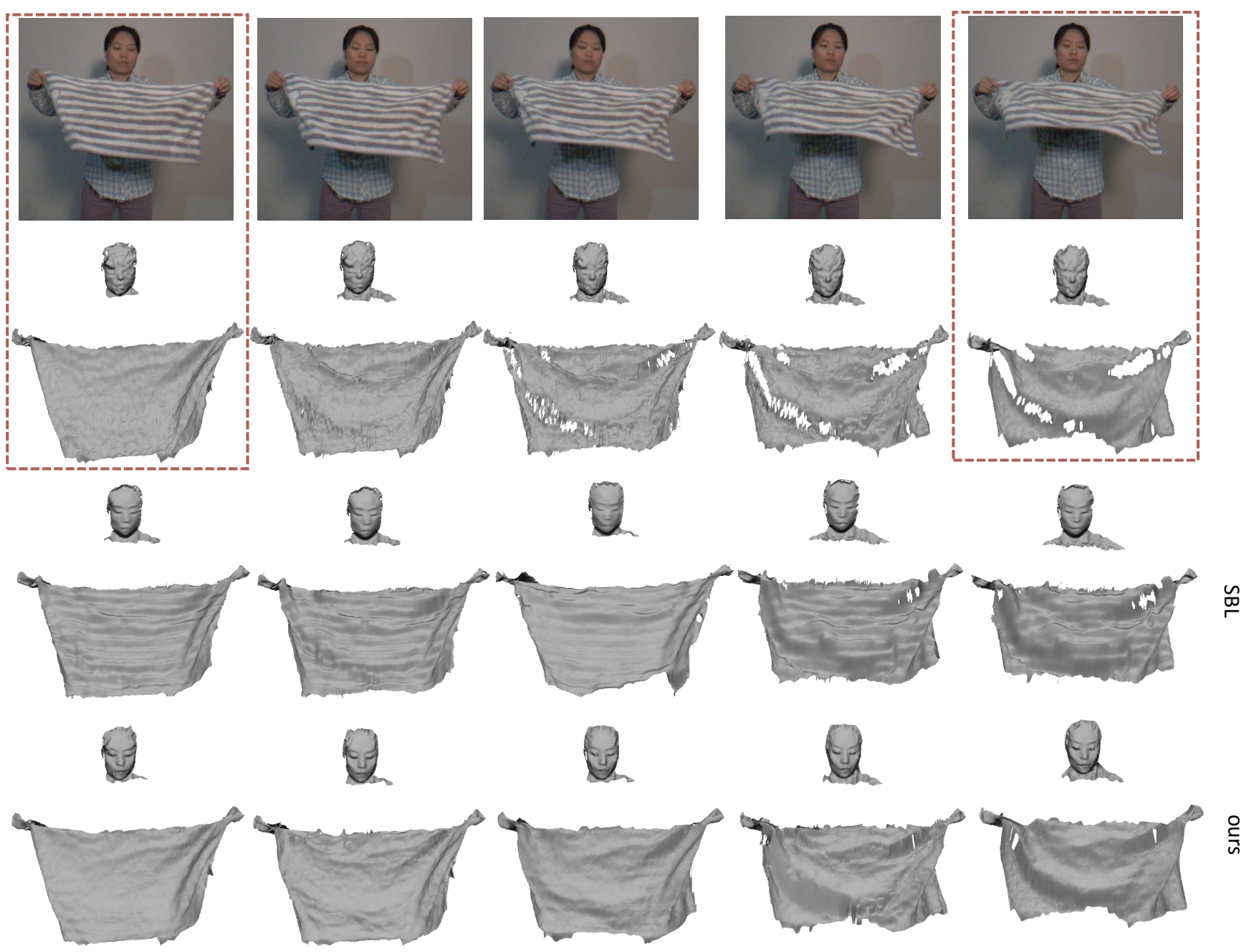

Figure 6: Results on the towel shaking sequence. To better demonstrate the shape of the towel, we adjust the perpective angle of the generated mesh which differs from the color image. First row shows the input color sequence with left most and right most have the corresponding input depth frame, while the middle ones are sampled frames between these two frames. Second row gives the input depth on the left and right, and interpolated depth with BL method for the middle frames. Third row displays the shading refinement result using SBL method. The last row shows the recovered depth from our method.

method yield better results thanks to the smooth velocity field constraints in the whole sequence and our bundle optimization approach. Also, considering the motion blur effect along the moving boundary, for the key frame depth, we detect the object boundaries with both depth and color contrast and enforce the gradient smoothness in the boundary area along with shading refinement. The refined key frame meshes are shown in (d) and (h).

Figure 6 shows the sequence where a towel is waved in front of the hybrid camera. For this case, the towel is experiencing both global translation and local deformations, which are more challenging to recover. Similar to the above case, the error in the interpolated depth will pass to the shading refinement. Also, the texture-copy problem has not been handled properly for the SBL method, as the stripe patterns on the towel are visible as hallucinated surface details. As most of the texture details have been separated clearly with our intrinsic decomposition, the recovered depth from our method is more faithful with the real surface details preserved.

More results can be seen in the supplementary material.

\subsection{Quantitative evaluation}

Quantitative evaluation for Synthetic data We use synthetic data to demonstrate the effectiveness of the global optimization framework for depth map generation compared to frame-by-frame method. The shading images are supposed to be given for the optimization framework, therefore we ignore the influence of albedo or texture.

We have generated two cases of synthetic data displayed in Figure 7 and Figure 8. For each sequence, it consists of seven depth frames with resolution of $64 \times 64$. We have the ground-truth lighting condition, therefore we can render the shading images for each frame. The optical flow between any frames are all set to zeros for simplicity, which means that the motion is purely in the $z$-direction. Given the first and last depth frame as two key frames and the shading 


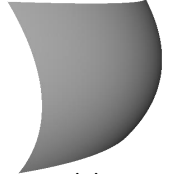

(a)

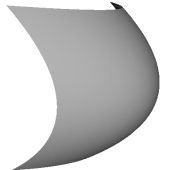

(e)

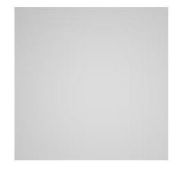

(b)

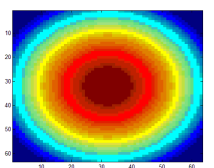

(f)

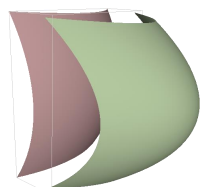

(c)

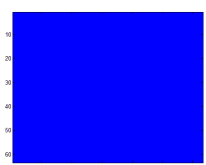

(g)

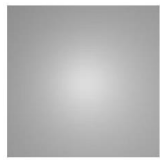

(d)

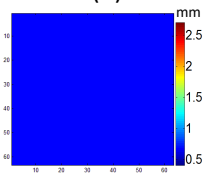

(h)
Figure 7: Results on synthetic data (simple case).

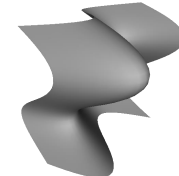

(a)

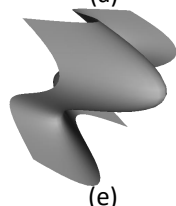

(e)

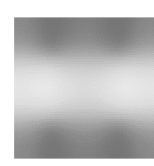

(b)

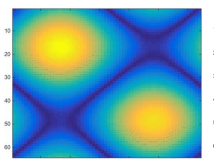

(f)

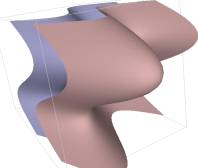

(c)

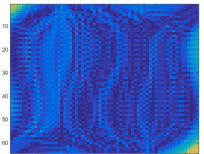

(g)

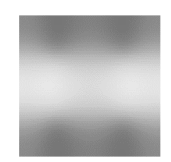

(d)

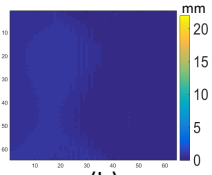

(h)
Figure 8: Results on synthetic data (more complex case). The two shading images (b) and (d) are the same to each other as we set the sequence to have half period of sine wave and these two key frames have the same shape, but they have the global displacement in depth, as shown in (c).

sequence, we present the result computed with BL, SBL, and also our method.

The first case (Figure 7) displays a simple case where the surface is bending forward along with the global linear translation. As illustrated in Figure 7, both SBL and our approach can recover the depth with quite small error.

For the second case (Figure 8), we have generated the depth sequence that has the sine wave in the shape deformation and also the global translation, which is the complex combination of linear and non-linear motion. For this more complex motion, our method will get smaller error than the BL and SBL method. The regularization terms and the global optimization will make our method converge to a better local minimum that is closer to the real depth.

For Figure 7 (Figure 8), the first row shows the mesh and shading image, which are the key frames as our input. Figure $7 \mathrm{a}$ (Figure 8a) is the mesh for the first frame with the shading image shown in Figure $7 \mathrm{~b}$ (Figure $8 \mathrm{~b}$ ). We put the meshes of the key frames together in Figure 7c (Figure 8c). The second row shows one sampled frame, where the first column Figure 7e (Figure 8e) is the groundtruth mesh and the next three columns present the error map for the depth maps recovered with BL, SBL and also our method respectively.

To better illustrate the results, we compute the relative depth error with the formula below, where the error is evaluated using the difference between recovered depth and the ground-truth depth divided by the displacement from the first frame,

$$
e r r=\frac{1}{T-1} \sum_{t=1}^{T-1} \frac{\sum_{p}\left|D^{t}(p)-R^{t}(p)\right|}{\sum_{p}\left|D^{t}(p)-D^{0}(p)\right|}
$$

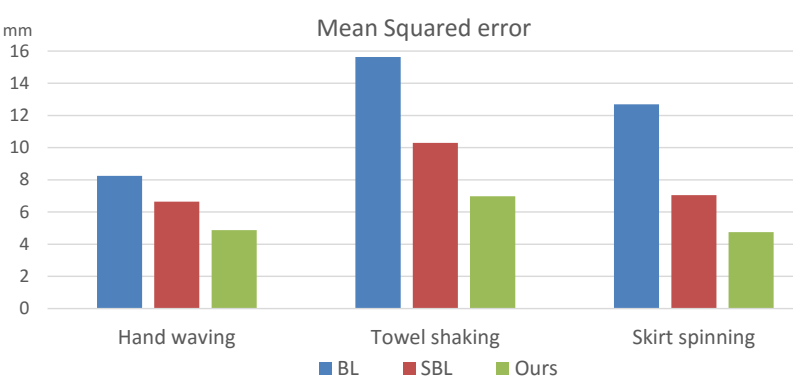

Figure 9: Quantitative results for real datasets. All the results are computed with squared mean error. We discard the extreme outliers around the surface boundaries during the evaluation.

For the BL method, the relative error is 0.1962 for the simple case and 0.3788 for complex case. The error will decrease to 0.0012 after the shading refinement (SBL) for the simple case and 0.0837 for the complex one. For our proposed method, the error is 0.0012 and 0.0569 respectively. Quantitative evaluation for real data To better validate our method on the real datasets with some quantitative measurement, we sample several frames from our real datasets and down-sample the captured depth frames into $15 \mathrm{~Hz}$ and then use these frames to recover all the depth sequences corresponding to color frames $(180 \mathrm{~Hz})$, which means for every two key frame depth maps, we will get thirteen depth frames interpolated from the interpolation methods. The middle frame corresponds to the original depth frames in $30 \mathrm{~Hz}$. The captured depth frames can be approximated as the groundtruth depth to measure the accuracy of the interpolated depth maps. We evaluate the BL method, SBL method and our approach using this strategy on three real datasets we have captured. We evaluate the performance of each method by measuring the mean value of the depth error compared to ground-truth depth. The results are shown in Fig. 9. Again our method is the best one.

Limitations During our experiments we have found a few limitations of our method. First, there are still some artifacts at the discontinuous boundary of the recovered depth which is caused by the motion blur effect of the captured depth sequence. This might be resolved using the normal constraints along the contours on the boundary. Secondly, we have not dealt with the occlusions and topology changes explicitly in our formulation, the artifacts exist in our recovered depth caused by the occlusions and topology changes. In future work, we plan to detect the occlusions via bi-directional matching and track the pixel or $3 \mathrm{D}$ point in the whole sequence and try to deal with the occlusions and topology changes.

\section{CONCLUSION}

In this paper, we present our novel hybrid camera setup, which is composed of a high-speed color camera and a depth sensor. Using this hybrid camera, we propose a framework to recover the depth sequence of the scene with high-speed motion. Considering that the bi-directional interpolation method will fail when there exists non-linear motion in the scene, we exploit the shading constraints in each color frame to overcome this limitation. In our formulation, we use the depth information captured from low-speed depth camera as the boundary constraints for the whole sequence and en- 
force the SfS constraints in each frame. Also, the depth maps of neighboring frames are associated with our proposed velocity term that preserves smooth motion field. Therefore, we can recovery the depth sequence in a single optimization. Finally, we present the comparison results for real datasets captured with our hybrid camera and also for synthetic datasets. Our high-speed and high-quality RGB-D sequence can be used in many areas where the motion is fast, such as sport event, gait analysis, etc.

\section{Acknowledgments}

The work is supported in part by US National Science Foundation grants IIS-1231545 and IIP-1543172, Natural Science Foundation of China (No.61332017, 61572243), Key Industrial Innovation Chain of Shaanxi Province Industrial Area (2015KTZDGY04-01), Chinese Scholarship Council, and Zhejiang Provincial Natural Science Foundation grant LY13F020019. Jiangbin Zheng and Ruigang Yang are the co-corresponding authors for this paper.

\section{REFERENCES}

[1] R. Achanta, A. Shaji, K. Smith, A. Lucchi, P. Fua, and S. Susstrunk. Slic superpixels compared to state-of-the-art superpixel methods. IEEE TPAMI, 34(11):2274-2282, 2012.

[2] O. M. Aodha, N. D. Campbell, A. Nair, and G. J. Brostow. Patch based synthesis for single depth image super-resolution. In $E C C V$, pages 71-84, 2012.

[3] J. T. Barron and J. Malik. High-frequency shape and albedo from shading using natural image statistics. In CVPR, pages 2521-2528, 2011.

[4] J. T. Barron and J. Malik. Shape, illumination, and reflectance from shading. IEEE TPAMI, 37(8):1670-1687, 2015.

[5] J. T. Barron and J. Malik. Intrinsic scene properties from a single rgb-d image. IEEE TPAMI, 38(4):690-703, 2016.

[6] H. G. Barrow and J. M. Tenenbaum. Recovering intrinsic scene characteristics from images. Comput. Vis. Syst., 1978.

[7] S. Bi, X. Han, and Y. Yu. An 11 image transform for edge-preserving smoothing and scene-level intrinsic decomposition. ACM Trans. Graph., 34(4):78, 2015.

[8] N. Bonneel, K. Sunkavalli, J. Tompkin, D. Sun, S. Paris, and H. Pfister. Interactive intrinsic video editing. $A C M$ Trans. Graph., 33(6):197, 2014.

[9] A. Bousseau, S. Paris, and F. Durand. User-assisted intrinsic images. ACM Trans. Graph., 28(5):130, 2009.

[10] T. Brox and J. Malik. Large displacement optical flow: descriptor matching in variational motion estimation. IEEE TPAMI, 33(3):500-513, 2011.

[11] Q. Chen and V. Koltun. A simple model for intrinsic image decomposition with depth cues. In ICCV, pages 241-248, 2013.

[12] J. Durou, M. Falcone, and M. Sagona. Numerical methods for shape-from-shading: A new survey with benchmarks. CVIU, 109(1):22-43, 2008.

[13] D. Ferstl, C. Reinbacher, R. Ranftl, M. RÃijther, and H. Bischof. Image guided depth upsampling using anisotropic total generalized variation. In $I C C V$, pages 993-1000, 2013.

[14] E. Garces, A. Munoz, J. Lopez-Moreno, and D. D. Gutierrez. Intrinsic images by clustering. In Computer Graphics Forum, volume 31, pages 1415-1424, 2012.

[15] Y. Han, J. Lee, and I. Kweon. High quality shape from a single rgb-d image under uncalibrated natural illumination. In $I C C V$, pages 1617-1624, 2013.
[16] S. M. Haque, A. Chatterjee, and V. M. Govindu. High quality photometric reconstruction using a depth camera. In CVPR, pages 2283-2290, 2014.

[17] E. Herbst, X. Ren, and D. Fox. Rgb-d flow: Dense 3-d motion estimation using color and depth. In ICRA, pages 2276-2282, 2013.

[18] B. K. P. Horn. Shape from Shading: A Method for Obtaining the Shape of a Smooth Opaque Object from One View. PhD thesis, MIT, 1970.

[19] J. Jeon, S. Cho, X. Tong, and S. Lee. Intrinsic image decomposition using structure-texture separation and surface normals. In ECCV, pages 218-233, 2014.

[20] N. Kong, P. Gehler, and M. Black. Intrinsic video. In ECCV, pages 360-375. 2014.

[21] P. Laffont, A. Bousseau, S. Paris, F. Durand, and G. Drettakis. Coherent intrinsic images from photo collections. ACM Trans. Graph., 31(6), 2012.

[22] E. H. Land and J. J. McCann. Lightness and retinex theory. JOSA, 1971.

[23] K. J. Lee, Q. Zhao, X. Tong, M. Gong, S. Izadi, S. U. Lee, P. Tan, and S. Lin. Estimation of intrinsic image sequences from image+ depth video. In ECCV, pages 327-340. 2012.

[24] R. Or-El, G. Rosman, A. Wetzler, R. Kimmel, and A. M. Bruckstein. Rgbd-fusion: Real-time high precision depth recovery. In $C V P R$, pages 5407-5416, 2015.

[25] J. Park, H. Kim, Y. Tai, M. S. Brown, and I. Kweon. High quality depth map upsampling for 3d-tof cameras. In ICCV, pages 1623-1630, 2011.

[26] R. Ramamoorthi and P. Hanrahan. An efficient representation for irradiance environment maps. In $A C M$ SIGGRAPH, pages 497-500, 2001.

[27] C. Richardt, C. Stoll, N. A. Dodgson, H. Seidel, and C. Theobalt. Coherent spatiotemporal filtering, upsampling and rendering of rgbz videos. In Computer Graphics Forum, volume 31, pages 247-256, 2012.

[28] J. Shi, Y. Dong, X. Tong, and Y. Chen. Efficient intrinsic image decomposition for rgbd images. In $A C M V R S T$, pages $17-25,2015$.

[29] Y. Tai, H. Du, M. S. Brown, and L. Stephen. Correction of spatially varying image and video motion blur using a hybrid camera. IEEE TPAMI, 32(6):1012-1028, 2010.

[30] M. F. Tappen, W. T. Freeman, and E. H. Adelson. Recovering intrinsic images from a single image. IEEE TPAMI, 27(9):1459-1472, 2005.

[31] C. Wu, K. Varanasi, Y. Liu, H. Seidel, and C. Theobalt. Shading-based dynamic shape refinement from multi-view video under general illumination. In $I C C V$, pages 1108-1115, 2011.

[32] C. Wu, M. Zollhöfer, M. NieÃßner, M. Stamminger, S. Izadi, and C. Theobalt. Real-time shading-based refinement for consumer depth cameras. ACM Trans. Graph., 33(3), 2014.

[33] Q. Yang, R. Yang, J. Davis, and D. Nistér. Spatial-depth super resolution for range images. In $C V P R, 2007$.

[34] G. Ye, E. Garces, Y. Liu, Q. Dai, and D. Gutierrez. Intrinsic video and applications. ACM Trans. Graph., 33(4):80, 2014.

[35] L. Yu, S. Yeung, Y. Tai, and S. Lin. Shading-based shape refinement of rgb-d images. In CVPR, pages 1415-1422, 2013.

[36] Q. Zhang, M. Ye, R. Yang, Y. Matsushita, B. Wilburn, and H. Yu. Edge-preserving photometric stereo via depth fusion. In $C V P R$, pages 2472-2479, 2012.

[37] Q. Zhao, P. Tan, Q. Dai, L. Shen, E. Wu, and S. Lin. A closed-form solution to retinex with nonlocal texture constraints. IEEE TPAMI, 34(7):1437-1444, 2012.

[38] J. Zhu, L. Wang, R. Yang, J. Davis, and Z. Pan. Reliability fusion of time-of-flight depth and stereo geometry for high quality depth maps. IEEE TPAMI, 33(7):1400-1414, 2011. 